\title{
A Stochastic Model Providing a Rationale for Adjuvant Chemotherapy ${ }^{1}$
}

$$
\text { by }
$$

James R. Thompson

and

Barry W. Brown

Technical Report 86-19, August 1986

${ }^{1}$ This work was supported in part by the United States Army Research Office (Durham) and the National Cancer Institute under DAAG 29-85-K-0212 and CA11430, respectively. 



\section{A Stochastic Model Providing a Rationale for Adjuvant Chemotherapy ${ }^{*}$}

James R. Thompson and Barry W. Brown

Rice University and M.D. Anderson Hospital \& Tumor Institute

Abstract A model yielding the probability of curative outcome for a patient at the time of tumor detection is presented. The status of the patient is determined by whether or not metastases (distant spread of the tumor) have occurred and whether any such metastases are drug resistant. If there are no metastases, then local excision is presumed curative; if there are nonresistant metastases, then local excision plus adjuvant chemotherapy is presumed curative; if any metastases are drug resistant, there is no cure. Metastases and drug resistance arise independently with intensities proportional to total tumor size. over a wide range of such intensities, the addition of adjuvant drug therapy yields a dramatic improvement in the probability of cure.

Keywords: adjuvant chemotherapy, resistance, metastasis

* This work was supported in part by the United States Army Research office (Durham) and the National Cancer institute under DAAG 29-85-K-0212 and CA1 1430, respectively. 
Introduction Goldie, Coldman and Gudauskas ([4],[5]) have explored the consequences of the buildup of resistance to one or more chemotherapeutic agents. They propose a model in which the probability of the emergence of a resistant cell at a given time is proportional to the size of the primary tumor at that instant. According to their model, resistance to a chemotherapeutic agent develops by mutation, randomly, spontaneously and independently of any application of that agent.

Another problem of interest is that of spread of the tumor to other sites through the process of metastatic progression [1]. Here, a cell may break of from the primary and move to another site in the body setting up a metastatic clone.

At the time of presentation, a patient with a solid tumor is in one of three fundamental states:

(a) no metastases

(b) metastases, none of which contain resistant cells

(c) metastases, at least one of which contains resistant cells.

Both simple excision of the primary and the adjuvant regime of excision plus chemotherapy will cure a patient in state (a). A patient in state (c) will not be cured by either regime. of special interest to us is the probability that, at the time of discovery (and removal) of the primary tumor the patient is in state (b). The probability that a patient has metastases, all nonresistant, at the time of presentation, gives us an indication as to the probability that a patient will be cured by adjuvant therapy but not by simple excision of the primary tumor. 
Discussion Following Goldie and Coldman [4], we shall assume all tumors grow according to
(1) $n(t)=e^{t}$,

where $n$ is the total number of tumor cells present.

The probability that a nonresistant tumor of size $n$ produces a resistant cell in the time interval $[t, t+\Delta]$ is given by

(2) P(resistant cell produced during $[t, t+\Delta])=B n(t) \Delta$, where $B$ represents the tendency to develop irreversible drug resistance.

Similarly, following Bartoszyński, Brown and Thompson [1], we shall assume that the probability a tumor of size $n(t)$ produces a metastasis in $[t, t+\Delta]$ is given by

(3) $P($ metastasis in $[t, t+\Delta])=\mu n(t) \Delta$, where $\mu$ represents the tendency to metastasize.

For $\mu$ and $\beta$ very small, we can use $n(t)$ as given simply by $e^{t}$, since here the amount of tumor mass removed from the primary to form the metastatic mass and/or the resistant mass is negligible. Furthermore, we assume that backwards mutation from resistance is negligible.

Let us consider the two events 
(4) $A(N)=$ the event that by the time the total tumor mass equals $\mathrm{N}$ cells a nonresistant metastasis develops in which a resistant population subsequently develops (before a total tumor mass of $\mathrm{N}$ ).

(5) $B(N)=$ the event that by the time the total tumor mass equals $\mathbf{N}$ cells a resistant clone develops from which a metastasis develops (before a total tumor mass of $\mathrm{N}$ ).

We shall seek to compute $P\left(A^{C}(N)\right)$ and $P\left(B^{C}(N)\right)$. Now,

(6) $P$ (metastasis occurs in $[t, t+\Delta]$ followed by a resistant subclone before $T)=\mu n\left[1-\exp \left(-\int_{0}^{T-t} B e^{\tau} d \tau\right)\right] \Delta$ $=\mu \mathrm{e}^{\mathrm{t}}\left[1-\mathrm{e}^{\beta} \exp (-\beta N / n)\right]$, where $T=\ln (N)$.

But then,

(7) $P\left(A^{C}(N)\right)=\exp \left[-\int_{0}^{T} \mu e^{t}\left(1-e^{B} \exp (-B N / n)\right] d t\right]$

$$
=\exp \left[\mu-\mu e^{B} e^{-B N}+\mu B e^{B} N(E i(-B)-E i(-B N)]\right]
$$
where the exponential integral $E i(x)$ is defined for negative $x$ by

(8) $\operatorname{Ei}(x)=-\int_{-x}-e^{-t / t} d t$. 
(9) $P\left[B^{C}(N)\right]=\exp \left[B-B e^{\mu} e^{-\mu N}+\mu B e^{\mu N} N\{E i(-\mu)-E i(-\mu N)]\right]$.

Thus, we can compute the probability that no resistant metastases have been formed by a total tumor size of $\mathrm{N}$ via

(10) $P($ no resistant metastases $)=P\left(A^{c}(N)\right) P\left(B^{C}(N)\right)$.

We have been able to obtain (7) and (9), exact solutions to the special two stage branching processes defined by hypotheses (1)-(3), (generally deemed nontractable and requiring approximation) by exploiting the special structure of the case of interest to us here: namely, the nonappearance of any second stage events. The exact expression in (10) is easily computed using, for example, the IMSL routine MMDEI [6] for computing exponential integrals. However for the magnitudes of $N$ (very large) and $\mu$ and $B$ (very small) in the present application, we obtain essentially the same result (using approximations [7] for small arguments of $E i(x)$ ) with

( 11 ) $P($ no resistant metastases by $N)=\left(\mu B \sigma^{2}\right)^{\mu B N}$ where $\delta=1.781072$ is e raised to Euler's constant.

The probability of no metastases at all by tumor size $\mathrm{N}$ is given simply by

(12) $P($ no metastases by $N)=\exp \left[-\int_{0}^{\top} \mu e^{t} d t\right]$

$$
=\exp [-\mu(N-1)] \text {. }
$$


Thus, the probability a patient with a tumor of size $\mathbf{N}$ is curable by adjuvant therapy but not by simple excision of the primary tumor alone is given by

(13) $\mathrm{P}$ (metastases, none of them resistant by $\mathrm{N})=$

$$
\left(\mu B \delta^{2}\right)^{\mu B N}-\exp [-\mu(N-1)] \text {. }
$$

In Figure 1, we show the probability of nonoccurrence of metastases versus $\log (\mu N)$. In Figure 2, we show the probability of nonoccurrence of resistant metastases versus $\log (N)$ for various values of $\log (\mu B)$. Typical values for $B$ for mammalian cells are $10^{-6}$ to $10^{-4}$ [4]. A typical tumor size at time of detection is $10^{10}$ cells (roughly 10 cubic centimeters). In Figure 3, we show the probability there exist metastases but no resistant metastases at a tumor mass of $10^{10}$ for various $B$ and $\mu$ values. We note, for example, that for $\beta=10^{-6.5}$ to $10^{-10}$, the probability a patient presents with a condition curable by adjuvant therapy but not by simple excision of the primary is at least $40 \%$. Similar results hold for early detection $\left(10^{9}\right.$ cells $)$ as shown in Figure 4 . This wide range of $\mu$ values includes that reported for breast cancer by Bartoszyński, Brown and Thompson [1].

An alternative procedure to that above can be based on an approximation used by Goldie, Coldman and Gudauskas in another context [5]. Using the axioms (1)-(3), letting $R(t)$ be the number of resistant cells, we may write

(14) $d E(R(t)) / d t=E(R)+\beta e^{t}=E(R)+\beta n(t)$

with solution 
(15) $F(R)=\beta n$ in $n$.

Then, the probability for no cells which develop first resistance and then metastasize is approximated by

(16) Pr[no metastases thrown of by resistant clones] $\approx$ $\exp \left(-\int_{0}^{\top} \mu E(R) d \tau\right]=\exp [-\mu \beta(1+N(\ln N-1])]$.

Similarly, we have that

(17) $\operatorname{Pr}[$ no metastasis which develops resistance] $\approx$ $\exp [-\mu B(1+N(\ln N-1))]$.

Then the $\mathrm{G}-\mathrm{C}-\mathrm{G}$ approximation to $(11)$ is given by

(18) $\operatorname{Pr}[$ no resistant metastasis by $N] \approx$ $\exp [-2 \mu B(1+N(\ln N-1])]$.

For probabilities greater than .5 , the $G-C-G$ approximation is very close to (11).

It has been reported [8] that in vivo $B$ values may actually be a hundred times greater than in vitro values. For $B$ values of .01 or greater, the assumption that the nonresistant population at time $t$ is given by $e^{t}$ is inappropriate. Accordingly, we consider below an approximation based on a modification of the G-C-G argument.

(19) $d(E(R)) / d t \approx E(R)+B\left[e^{t}-R\right]$

has the solution

(20) $E(R) \approx e^{t}-e^{t(1-\beta)}$.

Then,

(21) $\operatorname{Pr}[$ no metastasis thrown off by resistant clone by $N] \approx$ $\exp \left[-\mu(N-1)+\mu /(1-B)\left(N^{1-B}-1\right]\right]$.

Similarly,

(22) $\operatorname{Pr}$ [no metastasis thrown off which develops resistant clone by $N] \approx \exp \left[-B(N-1)+B /(1-\mu)\left(N^{1-\mu_{-}}-1\right]\right]$. 
Summarizing,

(23) Prino resistant metastasis by total tumor mass $\mathrm{N}] \approx$ $\exp \left[-(B+\mu)(N-1)+\mu /(1-B)\left(N^{(1-B)}-1\right)+B /(1-\mu)(N(1-\mu)-1)\right]$.

By differentiating (21) and (22) with respect to $N$, we note that for $B$ very large relative to $\mu$, the chances are that any formation of a resistant metastasis after tumor discovery would most likely be the result of spread from a resistant clone in the primary. Here, the standard protocol of removing the primary before beginning chemotherapy would be indicated. On the other hand, if the force of metastasis if much stronger than that of mutation to resistance, then chemotherapy might appropriately precede surgical intervention.

In the case of breast cancer which has metastasized at least to local nodes, it has been reported [2] that the use of adjuvant chemotherapy decreases disease mortality by as much as $54 \%$ when compared to surgery alone. In order to estimate $\mu$ and $B$ clinically, we need randomized trials on tumors (which have exhibited no metastases at presentation) using surgery followed by adjuvant chemotherapy compared with surgery alone. Such a clinical data base is not currently available. However, since we are, at this stage, really seeking rough estimates of $\mu$ and $B$, animal experiments may be appropriate.

We examine below how such experiments might be used to estimate $\mu$ and $B$. Let us suppose we have stratified our data by tumor size at presentation. Consider the $10^{10}$ primary cell stratum. Suppose that the control group (surgical excision only) exhigits a ture fate of $5 \pi$ and that the adjovant therapy group exhibits a $95 \%$ cure rate. Then we can estimate $\mu$ from (12):

(24) $\exp [-\hat{\mu}(N-1)]=\exp \left[-\hat{\mu}\left(10^{10}-1\right)\right]=.05$. 
This yields a in value of $3 \times 10^{-10}$.

Next, we can estimate $B$ from (11):

(25) $\left(\dot{\mu} \bar{\beta} \gamma^{2}\right)^{\mu \hat{\mu}}=\left(3 \times 10^{-10} \hat{\beta} \gamma^{2}\right)^{3}=.95$.

This gives (Using Newton's Method) $\hat{\beta}=.0006$. (The same estimate is obtained by the use of (23).)

Although we are here essentially concerned only with obtaining rough estimates of $\mu$ and $\beta$, it is clear that a number of resampling techniques (e.g., the jackknife or the bootstrap [3]), can be used to determine the variability of the estimates. Let us suppose, for example, that we have $N_{1}$ individuals in the control group of whom $n_{1}$ are cured and $N_{2}$ individuals in the adjuyant group of whom $n_{2}$ are cured. Using the coding of 1 for a cure and 0 for a noncure, we repeatedly sample (say $M$ times, where $M$ is very large) $N_{1}$ individuals $w$ ith probability of success $n_{1} / N_{1}$ and $N_{2}$ individuals with probability of success $n_{2} / N_{2}$. For each such $j$ 'th sampling we obtain $\dot{\mu}_{j}$ and $\dot{\vec{b}}_{j}$ as above. Then we have as ready bootstrap estimates for Var(j), $\operatorname{Var}(\hat{\beta})$, and $\quad \operatorname{Cov}(\dot{\mu}, \hat{\beta}), \quad \sum\left(\hat{\mu}_{j}-\bar{\mu}\right)^{2} / M, \quad \sum\left(\hat{\beta}_{j}-\bar{\beta}\right)^{2} / M \quad$ and $\sum\left(\dot{\mu}_{j}-\bar{\mu}\right)\left(\hat{\beta}_{j}-\bar{B}\right) / M$, respectively.

Conclusions He have developed a model which gives a framework in which the potential benefits of adjuvant chemotherapy can be considered. There seems to be a strong indication from the model that chemotherapy, in conjunction with surgery, is frequently preferred to surgery alone, not simply on considerations of palliation, but on considerations of cure. 


\section{References}

[1] Bartoszyński, Robert; Brown, Barry w. and Thompson, James R. (1982). "Metastatic and systemic factors in neoplastic progression," in Probability Models and Cancer , LeCam, Lucien and Neyman, Jerzy, eds., North Holland,253-264, 283-285.

[2] Buzdar, A. U, Hortobagyi, G.N., Marcus, C.E., Smith, T.L., Martin, R. and Gehan, E.A. (1986). "Results of adjuvant chemotherapy trials in breast cancer at M.D. Anderson Hospital and Tumor Institute." NCI Monographs, no. 1, 81-85.

[3] Efron, Bradley (1979). "Bootstrap methods--another look at the jackknife: Annals of Statistics, v. 7, 1-26.

[4] Goldie, J.H. and Coldman, A.J. (1979). "A mathematical model for relating the drug sensitivity of tumors to their spontaneous mutation rate." Cancer Treatment Reports, v. 63, 1727-1733.

[5] Goldie, J.H., Coldman, A.J. and Gudauskas, G. A. (1982).

"Rationale for the use of alternating non-cross-resistant chemotherapy." Cancer Treatment Reports, v.66, 439-449.

[6] IMSL, Inc. (1985). Subrout ine MMDEI.

[7] Jahnke, Eugene and Emde, Fritz (1945). Tables of Functions. Dover Publications, 1-3.

[8] Schimke, Robert T. (1984). "Gene amplification, drug resistance, and cancer." Cancer Research, v. 44, 1735-1742. 
Nonoccurrence of Metastases

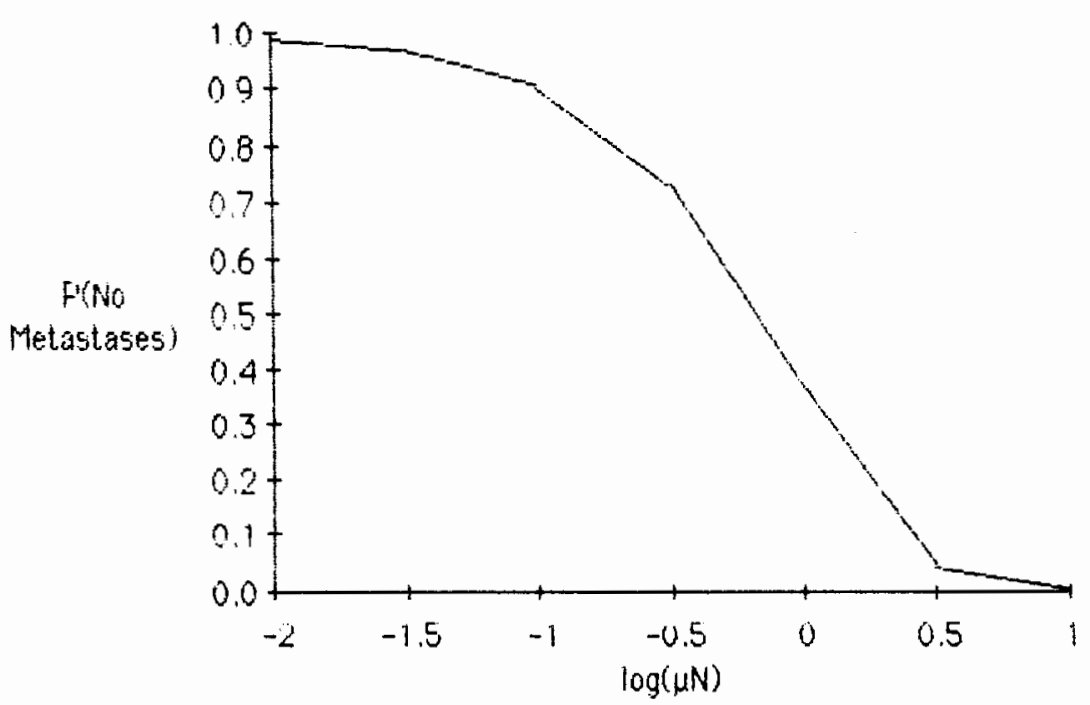

Fiqure 1 
Nonoccurrence of Resistant Metastases

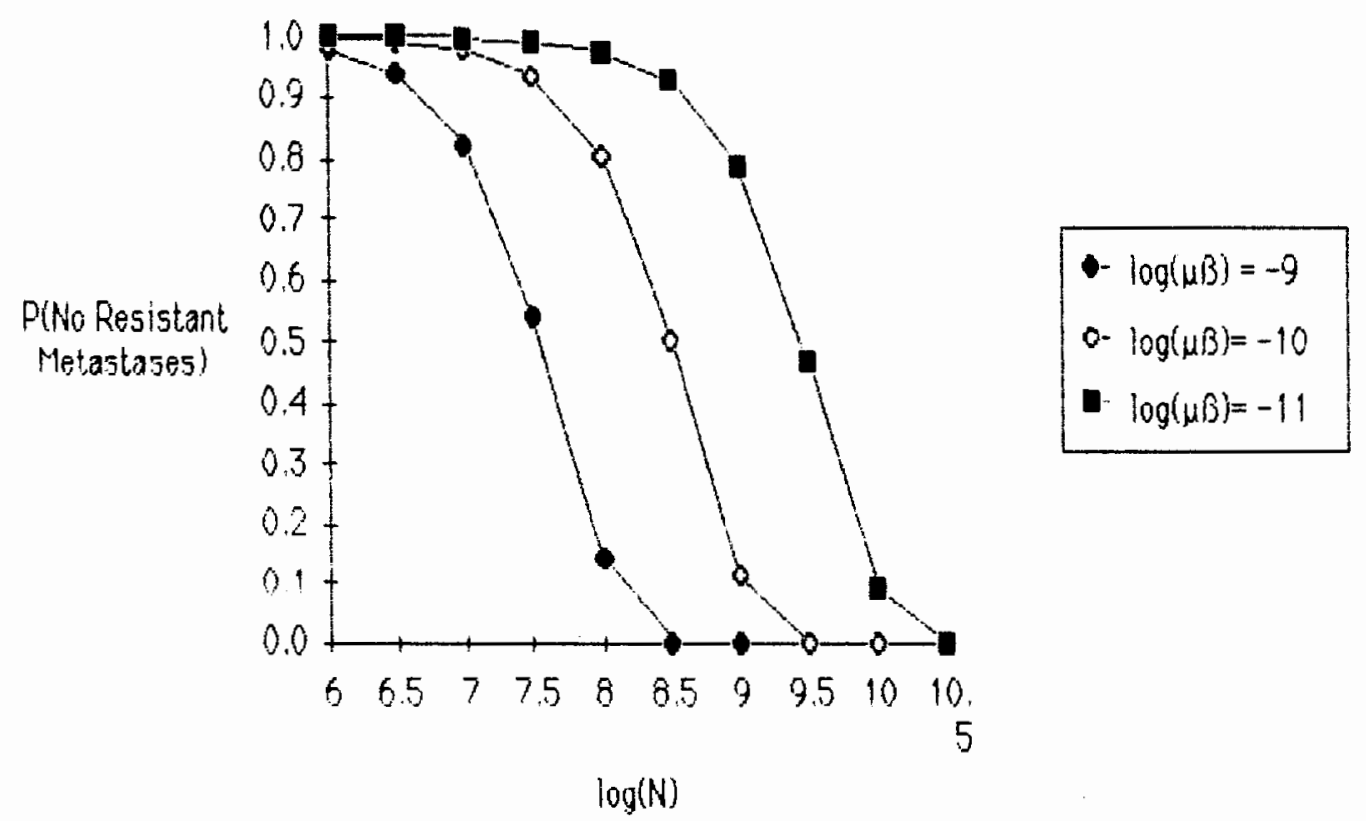

Figure 2 
Probability of Metastases But No Resistant Metastases When Log(Tumor Cell Count $)=10$

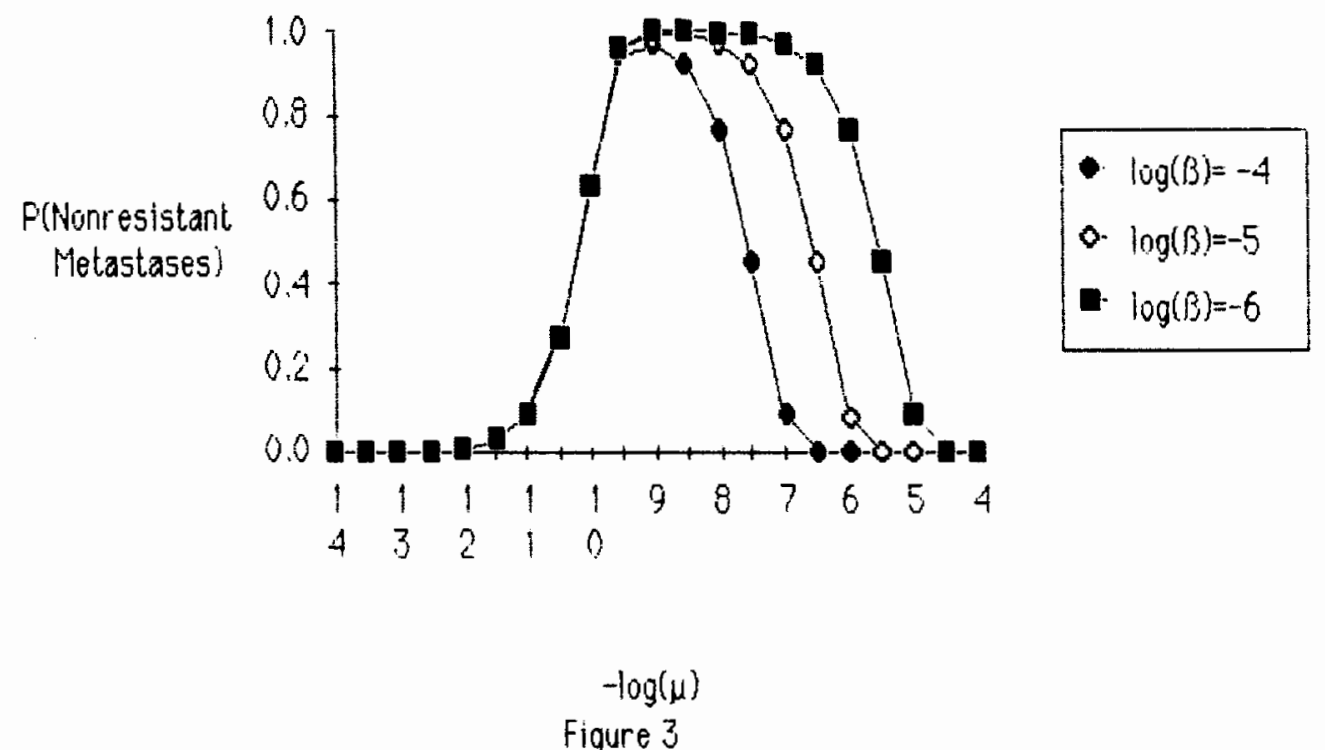

Figure 3 
Probatility There Esist Metastases But No

Resistant Metastases When Log(Tumor Cell

$$
\text { Couril) }=9
$$

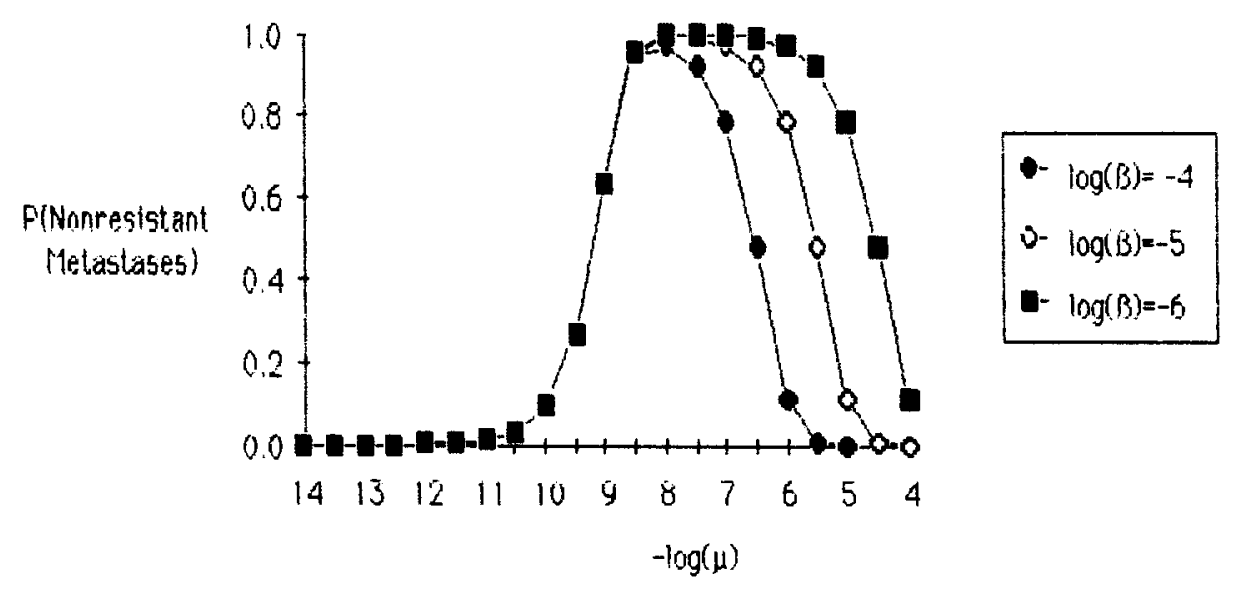

Figure 4 